\title{
FACTORES DE RIESGO PARA LA INCIDENCIA DE MASTITIS CLÍNICA EN GANADO LECHERO DE COSTA RICA
}

\author{
María Gabriela Mora*, Bernardo Vargas ${ }^{1 / *}$, Juan José Romero*, Jorge Camacho* \\ Palabras clave: Factor de riesgo; mastitis clínica; regresión logística; vacas lecheras. \\ Keywords: Fisk factors; clinical mastitis; logistic regression; dairy cows.
}

Recibido: 05/03/15

\section{RESUMEN}

Se realizó un estudio observacional retrospectivo para evaluar posibles factores de riesgo, relacionados con la vaca y su entorno, sobre la ocurrencia de primeros eventos de mastitis clínica en ganado lechero de Costa Rica. Se contó con información de 313406 lactancias, 101125 vacas y 288 hatos. La frecuencia relativa de mastitis a nivel poblacional fue de $11,6 \%$, con variaciones desde $0,3 \%$ hasta $70,7 \%$ entre los hatos. La tasa de incidencia poblacional fue de 4,65 casos por cada 10000 días a riesgo en la lactancia, con oscilaciones entre 0,092 y 5,7 para los diferentes hatos. Mediante regresión logística se evaluaron posibles factores de riesgo que afectan la incidencia de mastitis. Se exploraron 2 modelos mixtos lineales generalizados (GLMM), sin (modelo base) y con (modelo alternativo) efectos de lactancia previa. Los factores fijos con efecto significativo fueron: zona agroecológica, grupo racial, año, número y mes de parto, periodo de lactancia, duración y producción de leche en lactancia previa e historial previo de mastitis. Las categorías de mayor vs. menor propensión a contraer mastitis fueron, respectivamente: zona bosque seco tropical (OR Odds ratio:11,03) vs. bosque húmedo tropical (OR:0,97), grupo racial Jersey $\times$ Pardo Suizo (OR:1,67) vs. Pardo Suizo (OR: 1), partos en años previos a 1995 (OR:2,19)

1 Autor para correspondencia. Correo electrónico: bernardo.vargas.leitón@una.cr
Aceptado: 08/06/15

\begin{abstract}
Risk factors for clinical mastitis in dairy cattle of Costa Rica. A retrospective observational study was conducted to evaluate risk factors related to the cow and its environment on the occurrence of early events of clinical mastitis in dairy cattle of Costa Rica. Data on 313406 lactations from 101125 cows and 288 herds was available. The relative frequency of mastitis at the population level was $11.6 \%$, ranging from $0.3 \%$ to $70.7 \%$ between herds. The population incidence rate was 4.65 cases per 10 000 days at risk in lactation, ranging from 0.092 to 5.7 between herds. Logistic regression was used to evaluate potential risk factors affecting incidence of clinical mastitis. Two generalized linear mixed models (GLMM) were explored, without (base model) and with (alternative model) effects from previous lactation. The fixed factors with significant effect were: agroecological zone, racial group, year, number and month of calving, stage of lactation, duration and milk production in previous lactation, and history of mastitis in previous lactation. Categories with higher vs. lower propensity to mastitis were, respectively: tropical dry forest (OR Odds ratio: 11.03) vs. tropical rainforest (OR: 0.97), breed type Jersey×Brown Swiss (OR: 1.67) vs. Brown Swiss (OR: 1), birth-year before 1995 (OR:2.19) vs. after
\end{abstract}

\footnotetext{
* Posgrado Regional en Ciencias Veterinarias Tropicales, Universidad Nacional, Costa Rica.
} 
vs. posteriores a 2010 (OR:1), número de parto 4 (OR:1,19) vs. primer parto (OR:0,54), mes de parto Marzo (OR:1,25) vs. Octubre (OR:0,95), periodo de lactancia 1-30 días (OR:104) vs. 391420 días $(\mathrm{OR}: 0,94)$. En covariables, un incremento de $30 \mathrm{~d}$ en longitud de la lactancia anterior se asoció con OR de 1,04 y un incremento de 1000 $\mathrm{kg}$ en producción en la lactancia previa se asoció con un OR de 1,17. Estos hallazgos pueden ser útiles para el desarrollo de protocolos preventivos enfocados a reducir la incidencia de mastitis en los grupos de mayor riesgo.

\section{INTRODUCCIÓN}

La mastitis es la inflamación del tejido mamario productor de leche que se produce cuando microorganismos patogénicos entran a través del esfínter del pezón, multiplicándose y a su vez producen toxinas perjudiciales para la glándula mamaria (Nielsen 2009). Esto tiene como resultado la infiltración de constituyentes sanguíneos, proteínas séricas, enzimas y sales en la leche, además de disminuir el contenido de lactosa, caseína, grasa y proteína. También se producen incrementos en el nivel de elementos indeseables como enzimas lipasas y plasminas, las cuales rompen las estructuras de grasa y caseína en la leche, respectivamente (Blowey y Edmondson 2010).

En Costa Rica la industria láctea bonifica hasta un 3\% sobre el precio por kilogramo de leche pagado a sus productores cuando su Conteo de Células Somáticas (CCS) está por debajo de

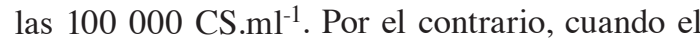

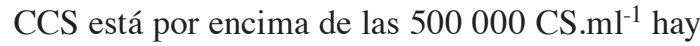
una reducción progresiva en el precio pagado por kilogramo de leche recibido, disminuido hasta un 20\%, cuando el CSS supera el $1000000{\mathrm{CS} . \mathrm{ml}^{-1}}^{-1}$ (Cooperativa de Productores de Leche Dos Pinos R.L. 2010).
2010 (OR: 1), fourth parity (OR:1.19) vs. first parity (OR: 0.54), month of calving March (OR: 1.25 ) vs. October (OR: 0.95), stage of lactation 1-30 days (OR:1.04) vs. 391-420 days (OR: 0.94). In covariates, an increase of $30 \mathrm{~d}$ in the previous lactation length was associated with an OR of 1.04 and an increase in production of $1000 \mathrm{~kg}$ in the previous lactation was associated with an OR of 1.17. These findings may be useful for the development of preventive protocols aimed at reducing the incidence of mastitis in groups with a higher risk.

La mastitis es una enfermedad de difícil erradicación ya que es causada por múltiples factores (Andresen 2001), asociados tanto a la vaca como a su entorno. Dentro de los factores asociados a la vaca se mencionan la raza, la predisposición genética, el número de partos o lactancias, la etapa de la lactancia, el nivel de producción y los intervalos reproductivos (Steeneveld et ál. 2008). Algunos de los factores del entorno que pueden influir en la incidencia de mastitis son la zona donde se ubica la finca, el hato-año-época de parto, las prácticas de alimentación, las prácticas del ordeño, la calidad e higiene del albergue, las condiciones climatológicas y las prácticas de manejo preventivo o terapéutico (Andresen 2001, Schukken et ál. 2010).

Una vaca puede presentar predisposición genética a contraer mastitis, asociada a determinadas características anatómicas, estado nutricional, parto, estado de lactación, involución mamaria, lactogénesis o el uso de determinados procedimientos de manejo (Rupp y Boichard 2004, Oltenacu y Broom 2010). Las vacas con altos registros de producción son más propensas a contraer mastitis, habiéndose reportado correlaciones genéticas de 0,45 entre producción e incidencia de mastitis clínica (Oltenacu y Broom 2010). 
Se ha reportado que las vacas más viejas tienden a presentar mayores CCS en la leche, mientras que las novillas de primer parto tienden a presentar CCS entre 20000 y 100000 (Blowey y Edmondson 2010). En lo que concierne a la etapa de la lactancia, se ha encontrado que durante el periodo seco existe mayor susceptibilidad a la mastitis clínica, principalmente 2 semanas después del secado y 2 semanas antes del parto (Biffa et ál. 2005, Elbably et ál. 2013).

Se ha encontrado que el clima y/o la época del año influyen sobre la presencia de mastitis. Es durante la estación lluviosa cuando existe una mayor proliferación y transmisión de patógenos, y por ende una mayor prevalencia de mastitis (Biffa et ál. 2005, Elbably et ál. 2013).

En Costa Rica, son pocos los estudios relacionados con esta enfermedad. Vargas et ál. (2013) reportaron un promedio de $365013{\mathrm{CS} . \mathrm{ml}^{-}}^{-}$ 1 en un grupo de 1081 fincas lecheras. Asimismo, Quesada (1994) encontró una prevalencia de mastitis subclínica de un $28 \%$ por vaca y del $11 \%$ por cuartos. No existe en el país ningún estudio a escala poblacional que evalúe posibles factores de riesgo que contribuyan en el desarrollo de la mastitis. El objetivo del presente estudio es estimar el efecto de factores de riesgo relacionados con la vaca, el hato y la zona agroecológica sobre la ocurrencia de primeros eventos de mastitis clínica durante la lactancia.

\section{MATERIALES Y MÉTODOS}

\section{Diseño y población}

Se realizó un estudio de carácter poblacional, observacional y retrospectivo, mediante el análisis de la información recolectada en la base nacional de datos VAMPP (Noordhuizen y Buurman 1984, Pérez et ál. 1989) del proyecto Consultoría Regional en Informática para la Producción Aninal Sostenible (CRIPAS), que cubre el periodo entre el 01/01/1985 y el 31/12/2013. La población estudiada estuvo constituida por 101 125 hembras procedentes de 288 hatos distribuidos en 9 zonas de vida. Un 95,5\% $(n=275)$ de los hatos corresponden a lecherías especializadas con ordeño mecánico y 2 ordeños diarios, con alimentación basada en pastoreo y suplementación con concentrado. El 4,5\% (n=13) restante de los hatos corresponden a sistemas de Doble Propósito con crianza de ternero al pie. No fue posible contar con información precisa sobre la rutina de ordeño practicada en dichos hatos.

Los productores lecheros ingresan voluntariamente la información de su animales (eventos productivos, reproductivos y sanitarios) al programa VAMPP. Esta información se envía periódicamente al proyecto CRIPAS, donde es sometida a revisión y edición para su análisis posterior. Los hatos participantes tienen diferentes periodos de seguimiento, que pueden variar desde pocos meses hasta más de 20 años. Asimismo, los periodos de seguimiento de las vacas y de las lactancias fueron de longitud variable.

\section{Definición de caso, criterios de inclusión y exclusión}

La información obtenida de los hatos es organizada a nivel de partos (lactancias) por vaca, de manera que cada parto representa una observación. Para el presente estudio el caso por analizar correspondió a la ocurrencia de primeros eventos de mastitis clínica dentro de las lactancias analizadas. Por lo tanto, cada lactancia en la que se reportó al menos un caso de mastitis clínica fue contabilizada como lactancia positiva, mientras que aquellas en las que no se reportó ningún caso se contabilizaron como negativas. En los casos de múltiples reportes de mastitis clínica dentro de una misma lactancia solo se contabilizó y analizó el primero.

Al ser un estudio observacional, el diagnóstico de mastitis clínica fue realizado por el personal de la finca basado en la observación de los síntomas característicos de la mastitis clínica (inflamación, dolor, secreción alterada de leche). Esto representa un factor importante de incertidumbre, ya que la eficiencia de registro y reporte de los eventos pudo variar sustancialmente entre hatos. No obstante, debe considerarse que la mastitis clínica además de ser de diagnóstico 
relativamente sencillo, es también una enfermedad que recibe especial atención en las lecherías especializadas, ya que impacta directamente la calidad del producto y existen penalizaciones importantes de parte de las plantas por la entrega de producto alterado (Cooperativa de Productores de Leche Dos Pinos R.L. 2010).

Como medida preventiva, se requirió que los hatos incluidos en el análisis tuvieran al menos 10 eventos positivos a mastitis clínica reportados, esto con el propósito de excluir los hatos que del todo no registran este tipo de evento sanitario.

\section{Análisis estadístico}

Se calcularon 2 estadísticos relacionados con la frecuencia de aparición del evento analizado. El primero fue la Frecuencia Relativa (FR) de mastitis clínica obtenido mediante la siguiente fórmula:

donde:

$$
F R=\frac{n_{(+)}}{N}
$$

$\mathrm{n}_{(+)}=$Número de lactancias positivas a mastitis clínica.

$\mathrm{N}=$ Número total de lactancias evaluadas.

Este parámetro es de fácil interpretación ya que simplemente refleja el porcentaje de lactancias afectadas dentro del total. Sin embargo, no es del todo eficiente para efectos comparativos, ya que las lactancias, animales y hatos presentaron diferentes periodos de seguimiento. Por este motivo se calculó también la tasa de incidencia (TI), (Rothman et ál. 2008), definida por la siguiente fórmula:

donde:

$$
T I=\frac{n_{(+)}}{\sum D A R}
$$

$\mathrm{DAR}=$ Número total de días a riesgo de sufrir el evento.

En el caso de lactancias completas, los DAR se obtuvieron como la longitud de la lactancia respectiva. En el caso de lactancias incompletas los DAR correspondieron a los días transcurridos desde el parto hasta el último día de lactancia registrado. En ambos casos, para lactancias demasiado extensas, se estableció un valor máximo de corte de 510 días. Los días posteriores a la ocurrencia de un evento de mastitis clínica no fueron contabilizados dentro de los DAR (Rothman et ál. 2008), por cuanto el evento analizado se define como primera ocurrencia de mastitis.

Se obtuvieron estadísticos de tendencia central y dispersión para FR y TI, tanto a nivel poblacional como por hato.

El análisis de los factores que afectan la ocurrencia de mastitis se realizó mediante un Modelo Mixto Lineal Generalizado (GLMM, Kleinbaum y Klein 2010), basado en una distribución logística. Se evaluaron 2 modelos: un modelo base y un modelo alternativo.

El modelo estadístico base se detalla seguidamente:

$$
\operatorname{LogitP}\left(F_{i j k t m n u}=1 x\right)=\left(\beta_{0}+v_{o i}\right)+\beta_{1} R_{j}+\beta_{2} E_{k}+\beta_{3} Y_{l}+\beta_{4} Z_{m}+\beta_{5} N_{n}+\beta_{6} E_{m}+h_{o u}+\varepsilon_{i j k l m n u}
$$

donde:

- Logit $\mathrm{P}\left(\mathrm{F}_{\mathrm{ijk} \mathrm{lmnu}}=1 \mathrm{X}\right)=$ Probabilidad de que el í-ésimo animal sufra un primer caso de mastitis clínica dado que pertenece a la j-ésima raza, pare en el k-ésimo mes, en el l-ésimo año, es de la m-ésima zona de vida, tiene n-ésimo número de partos y pertenece al u-ésimo hato. 
$\left(\beta_{0}+\mathrm{v}_{\mathrm{oi}}\right)=$ Intercepto sujeto-específico para el i-ésimo animal.

$\beta_{l} \mathrm{R}_{\mathrm{j}}=$ Efecto fijo del j-ésimo grupo racial. Clases: Holstein (H8), Jersey (J8), Pardo Suizo (PS8), Guernsey (G8), Cruce Holstein $\times$ Jersey $(\mathrm{H} \times \mathrm{J})$, Cruce Holstein $\times$ Pardo Suizo $(\mathrm{H} \times \mathrm{PS})$, Cruce Jersey $\times$ Pardo Suizo (J×PS), Cruce Bos taurus $\times$ Bos indicus $(\mathrm{BT} \times \mathrm{BI})$, Cruce Holstein $\times$ Pardo Suizo $\times$ Jersey $(\mathrm{H} \times \mathrm{P} \times \mathrm{J})$.

$\beta_{2} E_{k}=$ Efecto fijo para el k-ésimo mes de parto. Clases: Enero hasta Diciembre.

$\beta_{3} Y_{1}=$ Efecto fijo para el 1-ésimo año de parto. Clases: anterior a 1995, 1995 - 1999, $2000-2004,2005-2009$ y posterior a 2009. $\beta_{4} Z_{m}=$ Efecto fijo para la m-ésima zona de vida (Holdridge 1987). Clases: Bosque húmedo premontano, Bosque húmedo tropical, Bosque muy húmedo montano, Bosque muy húmedo montano bajo, Bosque muy húmedo premontano, Bosque muy húmedo tropical, Bosque pluvial montano bajo, Bosque seco tropical, Bosque húmedo montano bajo.

- $\quad \beta_{5} \mathrm{~N}_{\mathrm{n}}=$ Efecto fijo para el n-ésimo número de parto. Clases: 1 hasta $>=6$.

- $\quad \mathrm{B}_{6} \mathrm{E}_{\mathrm{o}}=$ Periodo de la lactancia. Clases: 1 a 30 días posparto, 31 a $60,(>=451$ días posparto).

- $\quad \mathrm{h}_{\mathrm{ou}}=$ Efecto aleatorio para el u-ésimo hato.

- $\quad \varepsilon=$ Error residual aleatorio $\mathrm{N}\left(0, \sigma_{\mathrm{s}}{ }^{2}\right)$.

El modelo alternativo consideró, además de los factores señalados para el modelo base, otros factores de riesgo adicionales relacionados con la lactancia previa, descritos seguidamente:

$\beta_{7} C_{p}=$ Efecto fijo para la o-ésima historia de casos de mastitis clínica previos (Clases: 1 Caso presente, 0 Caso no presente). $\mathrm{B}_{8} L_{q}=$ Efecto fijo para la q-ésima duración de la lactancia anterior (en días).

$\mathrm{B}_{9} S_{r}=$ Efecto fijo para la r-ésima duración del periodo seco (en días).

$\mathrm{B}_{10} P_{s}=$ Efecto fijo para la s-ésima producción previa corregida a 305 días (en $\mathrm{kg}$ ).
$\beta_{I l} A_{t}=$ Efecto fijo para la t-ésima duración del intervalo entre partos (en días).

En este modelo alternativo la cantidad de información analizada se redujo considerablemente, ya que se excluyeron las hembras de primer parto, que no tienen lactancia previa, y los hatos que no registran la producción de leche.

Ambos modelos fueron analizados mediante el procedimiento GLIMMIX del programa estadístico SAS (SAS Inst. 2009). Este procedimiento permite considerar modelos con efectos aleatorios, errores correlacionados y estructuras de correlación causadas por existencia de agrupaciones o conglomerados. En el presente estudio, los efectos de animal y hato fueron considerados como aleatorios, ya que un mismo animal pudo tener varios casos de mastitis durante su vida productiva. Asimismo, el efecto de hato puede causar una correlación estructural debido a que existen características afines dentro de ese conglomerado de animales.

A partir de los resultados del modelo se calcularon las probabilidades marginales (PM) de ocurrencia de mastitis clínica para cada categoría dentro de factor, así como también los cocientes de posibilidades (del inglés $O d d s$ Ratio, OR) de cada categoría con respecto a una categoría de referencia.

\section{RESULTADOS Y DISCUSIÓN}

\section{Frecuencia relativa y tasa de incidencia}

La base de datos editada se conformó por 101125 vacas distribuidas en 288 hatos lecheros. El total de lactancias analizadas fue de 313 406, para un promedio de 3,1 lactancias por vaca. Se contabilizó un total de 36415 lactancias con al menos 1 caso de mastitis clínica reportado, para una frecuencia relativa (FR) poblacional de $11,62 \%$. A nivel de hato las frecuencias relativas oscilaron desde un mínimo de $0,3 \%$ hasta un máximo de $70,7 \%$ con un promedio de $13,6 \pm 12,2 \%$ (Figura 1, superior). La frecuencia obtenida se asemeja a prevalencias reportadas 

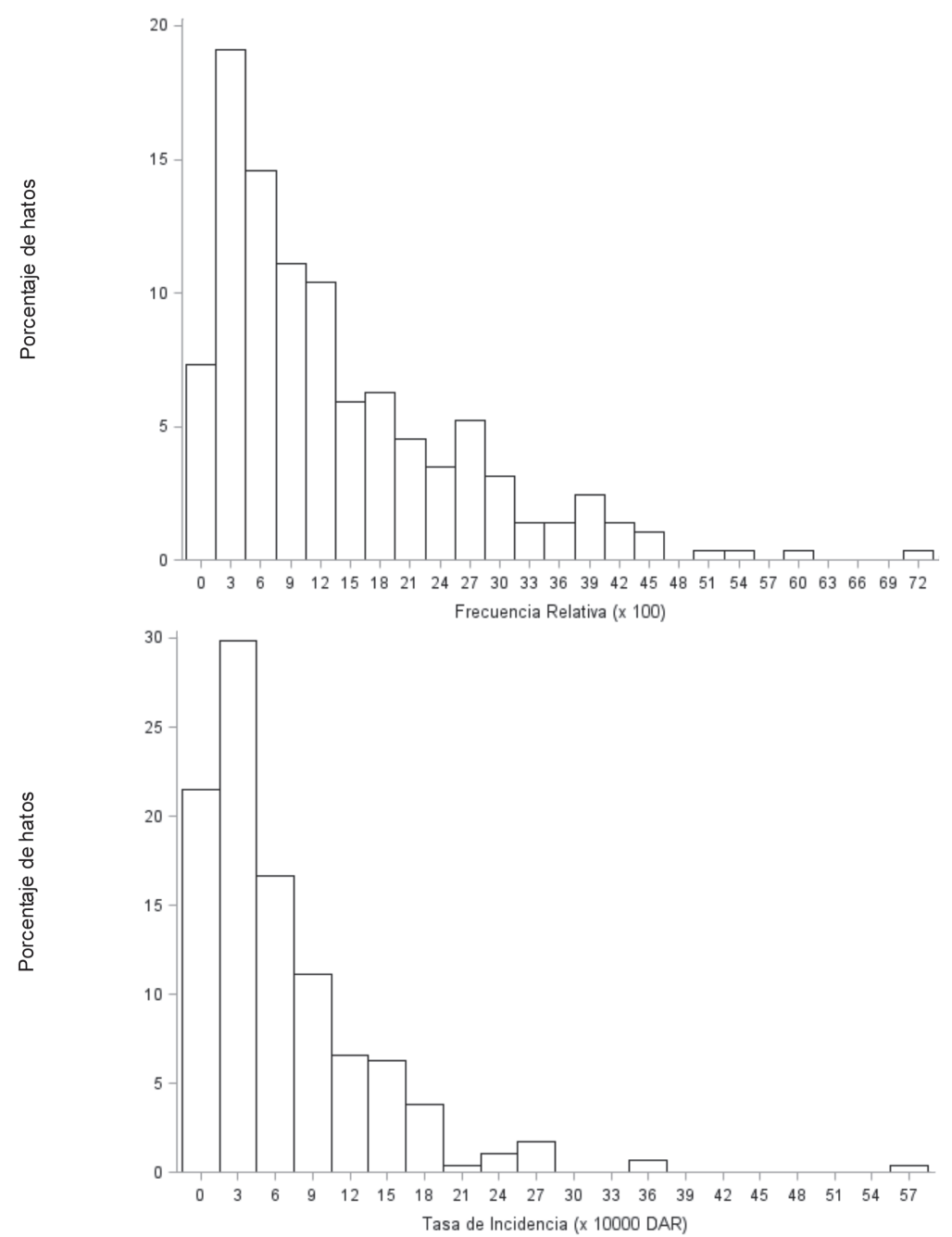

Fig. 1. Distribución de hatos según frecuencia relativa (superior) y tasa de incidencia (inferior) de mastitis clínica (n=288 hatos).

por algunos estudios, tales como 11,9\% (Biffa et ál. 2005) o 9,9\% (Elbably et ál. 2013). En otros estudios los valores reportados son mayores, tales como el $19,1 \%$ obtenido por Steeneveld et ál. (2008), mientras que en otros son menores, tales como el 1,7\% reportado por Ramírez et ál. (2011) o el 3,9\% reportado por Mekonen et ál. (2012). Olde Riekerink et ál. (2007) reportaron una incidencia de mastitis clínica de 23\% en 106 hatos de Canadá, que fluctuaron entre 7,6\% y $31,6 \%$, para diferentes regiones. Cabe señalar que en el presente estudio, a diferencia de otros, solo se contabilizaron los primeros casos de mastitis clínica en la lactancia.

La tasa de incidencia (TI) poblacional fue de 0,000465 por DAR, lo que equivale a 4,65 casos por cada 10000 DAR o 0,17 casos por año a riesgo. Steeneveld et ál. (2008) reportaron TI 
para mastitis en el rango entre 0,0002 y 0,0074 por DAR, similares al presente estudio. Olde Riekerink et ál. (2007) reportaron TI de 0,00063 casos de mastitis por DAR, ligeramente mayor que en el presente estudio. Otros estudios reportaron valores de 0,14 (Gianneechini et ál. 2002) y 0,21 (Almaw et ál. 2012) casos de mastitis por año a riesgo, también cercanos a los encontrados en el presente estudio.

A nivel de hato las TI oscilaron entre un mínimo de $9,2 \times 10^{-6}$ y un máximo de 0,0057 por DAR, con un promedio de $0,000657 \pm 0,000071$, equivalentes a 6,6 casos por cada 10000 DAR o 0,24 caso por año a riesgo (Figura 1, inferior). Olde Riekerink et ál. (2007) reportaron un rango de variación entre hatos para Ti desde $1,98 \times 10^{-5}$ hasta 0,0027 por DAR, similar al encontrado en el presente estudio.

En la Figura 1 se puede observar además que las distribuciones de FR y TI entre hatos son claramente sesgadas, ya que la mayoría de los hatos presenta bajas FR o TI, mientras que solo unos pocos se desvían a valores más elevados. Es importante considerar que existió una gran variación en la cantidad de lactancias disponibles por hato (40-16023), por lo que la precisión de los estimados intrahato fue muy heterogénea. Es posible también que se haya presentado un subregistro de casos de mastitis en algunos hatos, debido a que los datos fueron reportados voluntariamente.

\section{Modelos de regresión logística}

Ambos modelos de regresión logística convergieron exitosamente y con ajustes altamente significativos. Los resultados del modelo base indicaron que todos los factores de riesgo evaluados fueron altamente significativos $(\mathrm{p}<0,001) \mathrm{o}$ significativos ( $p<0,05$, Cuadro 1$)$. En el modelo

Cuadro 1. Grados de libertad, valor F y valor $\mathrm{P}$ asociados a factores riesgo que inciden sobre la ocurrencia de mastitis clínica en ganado lechero de Costa Rica, obtenidos del análisis de regresión logística de efectos mixtos en el modelo base y modelo alternativo.

\begin{tabular}{|c|c|c|c|c|c|c|}
\hline \multirow[b]{2}{*}{ Factores de riesgo } & \multicolumn{3}{|c|}{ Modelo base $^{1}$} & \multicolumn{3}{|c|}{ Modelo alternativo $^{2}$} \\
\hline & $\mathrm{GL}^{3}$ & Valor F & Valor $\mathrm{p}$ & GL & Valor F & Valor $\mathrm{p}$ \\
\hline \multicolumn{7}{|l|}{ Efectos Fijos } \\
\hline Tipo racial & 8 & 33,1 & $<0,001$ & 6 & 8,9 & $<0,001$ \\
\hline Mes de parto & 11 & 6,2 & $<0,001$ & 11 & 6,7 & $<0,001$ \\
\hline Año de parto & 4 & 188,3 & $<0,001$ & 4 & 155,6 & $<0,001$ \\
\hline Zona ecológica & 8 & 2,5 & 0,010 & 8 & 1,6 & 0,129 \\
\hline Número de parto & 5 & 242,1 & $<0,001$ & 4 & 35,1 & $<0,001$ \\
\hline Periodo de lactancia & 14 & 1930,1 & $<0,001$ & 14 & 1333,6 & $<0,001$ \\
\hline Caso previo de mastitis & - & - & - & 1 & 845,5 & $<0,001$ \\
\hline Duración de la lactancia previa ${ }^{4}$ & - & - & - & 1 & 97,9 & $<0,001$ \\
\hline Duración del periodo seco previo 4 & - & - & - & 1 & 3,8 & 0,051 \\
\hline Producción de leche (305 d) previa ${ }^{4}$ & - & - & - & 1 & 318,2 & $<0,001$ \\
\hline Efectos aleatorios & & Estim. & E.E & & Estim. & E.E \\
\hline Hato & & 1,4 & 0,11 & & 1,7 & 0,16 \\
\hline Residual & & 1,2 & 0,003 & & 1,2 & 0,004 \\
\hline
\end{tabular}

El modelo base analizó 313406 lactancias, de 101125 vacas en 288 hatos.

El modelo alternativo analizó 172346 lactancias, de 63304 vacas en 280 hatos.

Grados de libertad del efecto correspondiente.

Incluidas como covariable. 
alternativo la mayoría de los efectos también fueron altamente significativos $(\mathrm{p}<0,001)$, con excepción de zona ecológica y periodo de secado previo, los cuales no presentaron significancia. Los resultados para los factores de riesgo comunes a ambos modelos fueron en su mayoría consistentes en términos de significancia, aunque se observó una tendencia a menores valores de $\mathrm{F}$ en el modelo alternativo, lo que puede atribuirse en general al menor número de lactancias (y grados de libertad) disponibles en dicho análisis. $\mathrm{La}$ zona ecológica fue el factor que mostró un mayor cambio en significancia entre ambos modelos, pero aún así presentó patrones similares en los estimados de OR de las categorías comparadas.

Como se observa en el Cuadro 1, los componentes de varianza estimados para los efectos aleatorio de hato y residual fueron similares en ambos modelos, en ambos casos al ser mayor el componente del hato, lo que denota la importancia del factor hato en la variabilidad observada para la ocurrencia de mastitis, según se apreció también en la Figura 1. En el presente estudio no fue posible contar con información sobre las prácticas generales de manejo existentes en cada hato, entre ellas la rutina al momento del ordeño. Estas prácticas pueden contribuir en gran parte a la alta variabilidad que se observa en la Figura 1.

Los Cuadros 2 y 3 muestran los cocientes de posibilidades (OR) y las Probabilidades Marginales (PM) de presentación de primeros eventos de mastitis para vacas en diferentes categorías de un mismo factor de riesgo, obtenidos a partir de la solución del modelo alternativo. Los valores obtenidos del modelo base (no mostrados) fueron sumamente similares para las categorías coincidentes.

En el caso del tipo racial (Cuadro 2), los grupos más propensos a sufrir mastitis clínica fueron el cruce de Jersey $\times$ Pardo Suizo y la raza Guernsey, mientras que el grupo menos propenso fue la raza Pardo Suizo. Entre las 2 razas más comunes en Costa Rica, se observa que la Holstein tiende a una mayor propensión que la Jersey, si bien los OR no difieren significativamente entre sí. Las probabilidades de mastitis oscilan entre 0,07 (Pardo Suizo) y 0,11 (Jersey $\times$ Pardo Suizo y Guernsey). Los OR de los grupos Bos taurus $\times$ Bos indicus y el cruce Holstein $\times$ Pardo Suizo $\times$ Jersey no aparecen en este modelo alternativo, ya que no contaron con información para la covariable de producción. No obstante, los OR obtenidos para estos grupos según el modelo base fueron, respectivamente: 1,02 (IC95\% 0,90-1,16) y 1,36 (IC95\% 1,04-1,80).

En algunos estudios las vacas de la raza Holstein presentaron un riesgo mayor de sufrir mastitis, en comparación con vacas de las razas Jersey, Deoni, criollas, y sus cruces (Biffa et ál. 2005, Santivañez et ál. 2013). La selección intensa por parámetros de producción en algunas razas lecheras podría influir negativamente en su propensión a mastitis, dado que existe una correlación genética negativa entre producción y propensión a mastistis (Oltenacu y Broom 2010). Otros estudios no reportan efecto significativo de la raza sobre la prevalencia de mastitis (Rahman et ál. 2009, Prendiville et ál. 2010, Ramírez et ál. 2011, Richert et ál. 2013).

En cuanto al año de parto (Cuadro 2), la mayor propensión a mastitis se observó en las vacas paridas antes de 1995 y la menor en vacas paridas después del 2009. La tendencia en los periodos intermedios no es consistente, ya que se presentan altibajos. Las probabilidades marginales de mastitis oscilaron entre 0,06 (posterior a 2009) y 0,13 (anterior a 1995). Estas variaciones en el tiempo pueden estar ligadas a varios factores, tanto ambientales como de manejo. En primer lugar debe considerarse que la población de hatos no es uniforme, sino que ha ido en aumento con el paso del tiempo. Los factores climáticos también pueden sufrir cambios notables entre distintos años o periodos.

De acuerdo con el modelo alternativo la zona agroecológica (Cuadro 2) en la que se dio mayor propensión a mastitis fue el bosque tropical seco (bs_t) y las de menor propensión fueron el bosque húmedo tropical (bh_t) y el bosque húmedo montano bajo (bh_mb). Las probabilidades marginales de mastitis oscilaron entre 0,05 (bh_t) y 0,39 (bs_t). Aunque esta variable no fue 
Cuadro 2. Cociente de posibilidades (del inglés odds ratio, $\mathrm{OR}$ ) y probabilidad marginal (PM) a sufrir mastitis clínica con intervalos de confianza (IC95\%) para los factores de riesgo tipo racial, año de parto, zona ecológica y número de parto, según modelo alternativo.

\begin{tabular}{|c|c|c|c|c|c|c|c|}
\hline \multirow{2}{*}{ Factores de riesgo } & \multirow{2}{*}{$\mathrm{n}$} & \multirow{2}{*}{ OR } & \multicolumn{2}{|c|}{$\mathrm{IC}_{\mathrm{OR}} 95 \%$} & \multirow{2}{*}{ PM } & \multicolumn{2}{|c|}{$\mathrm{IC}_{\mathrm{PM}} 95 \%$} \\
\hline & & & LI & LS & & LI & LS \\
\hline \multicolumn{8}{|l|}{ Tipo racial } \\
\hline Jersey & 49429 & 1,38 & 1,17 & 1,63 & 0,09 & 0,07 & 0,12 \\
\hline Holstein $\times$ Jersey & 33968 & 1,35 & 1,15 & 1,58 & 0,09 & 0,07 & 0,11 \\
\hline Holstein & 70897 & 1,56 & 1,34 & 1,83 & 0,10 & 0,08 & 0,13 \\
\hline Holstein $\times$ Pardo Suizo & 7919 & 1,28 & 1,14 & 1,45 & 0,09 & 0,07 & 0,11 \\
\hline Jersey $\times$ Pardo Suizo & 2350 & 1,67 & 1,37 & 2,04 & 0,11 & 0,08 & 0,14 \\
\hline Guernsey & 1699 & 1,65 & 0,98 & 2,77 & 0,11 & 0,06 & 0,17 \\
\hline Pardo Suizo & 6084 & 1,0 & & & 0,07 & 0,05 & 0,09 \\
\hline \multicolumn{8}{|l|}{ Año de parto } \\
\hline Previo a 1995 & 11375 & 2,19 & 1,98 & 2,43 & 0,13 & 0,10 & 0,16 \\
\hline 1995 a 1999 & 27624 & 1,66 & 1,54 & 1,80 & 0,10 & 0,08 & 0,13 \\
\hline 2000 a 2004 & 37989 & 1,30 & 1,22 & 1,39 & 0,08 & 0,06 & 0,10 \\
\hline 2005 a 2009 & 50489 & 1,93 & 1,82 & 2,04 & 0,11 & 0,09 & 0,14 \\
\hline Posterior a 2009 & 44869 & 1,0 & & & 0,06 & 0,05 & 0,08 \\
\hline \multicolumn{8}{|l|}{ Zona ecológica } \\
\hline Bosque húmedo premontano & 26538 & 1,76 & 0,71 & 4,37 & 0,09 & 0,06 & 0,13 \\
\hline Bosque muy húmedo montano & 1364 & 1,28 & 0,28 & 5,91 & 0,07 & 0,02 & 0,21 \\
\hline Bosque muy húmedo montano bajo & 21282 & 1,69 & 0,66 & 4,28 & 0,09 & 0,06 & 0,13 \\
\hline Bosque muy húmedo premontano & 38886 & 1,64 & 0,69 & 3,91 & 0,09 & 0,06 & 0,12 \\
\hline Bosque muy húmedo tropical & 44156 & 1,39 & 0,59 & 3,30 & 0,07 & 0,05 & 0,10 \\
\hline Bosque pluvial montano bajo & 12275 & 1,77 & 0,69 & 4,50 & 0,09 & 0,06 & 0,14 \\
\hline Bosque seco tropical & 931 & 11,33 & 2,30 & 55,75 & 0,39 & 0,14 & 0,72 \\
\hline Bosque húmedo montano bajo & 20537 & 1,03 & 0,38 & 2,75 & 0,06 & 0,03 & 0,09 \\
\hline Bosque húmedo tropical & 6377 & 1,0 & & & 0,05 & 0,02 & 0,11 \\
\hline \multicolumn{8}{|l|}{ Número de parto } \\
\hline 2 & 46144 & 1,03 & 0,97 & 1,09 & 0,08 & 0,06 & 0,11 \\
\hline 3 & 38604 & 1,25 & 1,18 & 1,33 & 0,10 & 0,08 & 0,13 \\
\hline 4 & 30339 & 1,36 & 1,27 & 1,45 & 0,11 & 0,08 & 0,13 \\
\hline 5 & 22488 & 1,23 & 1,15 & 1,32 & 0,10 & 0,08 & 0,12 \\
\hline 6 y posteriores & 34771 & 1,00 & & & 0,08 & 0,06 & 0,10 \\
\hline
\end{tabular}


Cuadro 3. Cociente de posibilidades (del inglés odds ratio, OR) y probabilidad marginal (PM) de ocurrencia de mastitis clínica, con intervalos de confianza (IC95\%) para los factores de riesgo de lactancia, mes de parto y periodo de lactancia, según modelo alternativo.

\begin{tabular}{|c|c|c|c|c|c|c|c|}
\hline \multirow{2}{*}{ Factores de riesgo } & \multirow{2}{*}{$\mathrm{n}$} & \multirow{2}{*}{ OR } & \multicolumn{2}{|c|}{$\mathrm{IC}_{\mathrm{OR}} 95 \%$} & \multirow{2}{*}{ PM } & \multicolumn{2}{|c|}{$\mathrm{IC}_{\mathrm{PM}} 95 \%$} \\
\hline & & & LI & LS & & LI & LS \\
\hline \multicolumn{8}{|l|}{ Mes de Parto } \\
\hline Enero & 15294 & 1,03 & 0,94 & 1,13 & 0,09 & 0,07 & 0,11 \\
\hline Febrero & 12274 & 1,17 & 1,06 & 1,29 & 0,10 & 0,08 & 0,13 \\
\hline Marzo & 13133 & 1,25 & 1,13 & 1,38 & 0,10 & 0,08 & 0,13 \\
\hline Abril & 13029 & 1,24 & 1,12 & 1,36 & 0,10 & 0,08 & 0,13 \\
\hline Mayo & 13273 & 1,18 & 1,07 & 1,30 & 0,10 & 0,08 & 0,13 \\
\hline Junio & 12382 & 1,13 & 1,03 & 1,25 & 0,10 & 0,07 & 0,12 \\
\hline Julio & 12547 & 1,14 & 1,03 & 1,26 & 0,10 & 0,07 & 0,12 \\
\hline Agosto & 13869 & 1,04 & 0,94 & 1,14 & 0,09 & 0,07 & 0,11 \\
\hline Setiembre & 15706 & 1,06 & 0,97 & 1,16 & 0,09 & 0,07 & 0,11 \\
\hline Octubre & 17318 & 0,95 & 0,87 & 1,04 & 0,08 & 0,06 & 0,10 \\
\hline Noviembre & 16918 & 0,98 & 0,90 & 1,07 & 0,08 & 0,06 & 0,11 \\
\hline Diciembre & 16603 & 1,00 & & & 0,09 & 0,07 & 0,11 \\
\hline \multicolumn{8}{|l|}{ Periodo de Lactancia } \\
\hline 1 - 30 días posparto & 9249 & 103,98 & 89,89 & 120,27 & 0,63 & 0,57 & 0,69 \\
\hline 31-60 días posparto & 6578 & 41,52 & 35,81 & 48,13 & 0,41 & 0,34 & 0,48 \\
\hline 61 - 90 días posparto & 7360 & 31,11 & 26,84 & 36,05 & 0,34 & 0,28 & 0,40 \\
\hline 91- 120 días posparto & 7492 & 24,87 & 21,45 & 28,84 & 0,29 & 0,24 & 0,35 \\
\hline 121 - 150 días posparto & 7208 & 22,52 & 19,40 & 26,14 & 0,27 & 0,22 & 0,33 \\
\hline 151- 180 días posparto & 7183 & 18,59 & 16,00 & 21,59 & 0,23 & 0,19 & 0,29 \\
\hline 181 - 210 días posparto & 7337 & 14,54 & 12,50 & 16,91 & 0,19 & 0,15 & 0,24 \\
\hline $211-240$ días posparto & 8984 & 8,88 & 7,63 & 10,33 & 0,13 & 0,10 & 0,16 \\
\hline 241 - 270 días posparto & 16033 & 3,11 & 2,68 & 3,63 & 0,05 & 0,04 & 0,06 \\
\hline 271- 300 días posparto & 29425 & 1,13 & 0,97 & 1,31 & 0,02 & 0,01 & 0,02 \\
\hline 301 - 330 días posparto & 23073 & 0,97 & 0,83 & 1,14 & 0,02 & 0,01 & 0,02 \\
\hline $331-360$ días posparto & 14937 & 1,10 & 0,93 & 1,31 & 0,02 & 0,01 & 0,02 \\
\hline 361 - 390 días posparto & 9849 & 1,06 & 0,87 & 1,28 & 0,02 & 0,01 & 0,02 \\
\hline 391 - 420 días posparto & 6430 & 0,94 & 0,75 & 1,18 & 0,02 & 0,01 & 0,02 \\
\hline 421 - 450 días posparto & 11208 & 1,00 & & & 0,02 & 0,01 & 0,02 \\
\hline \multicolumn{8}{|l|}{ Mastitis en Lactancia Previa } \\
\hline Sí & 19764 & 2,10 & 2,00 & 2,21 & 0,13 & 0,10 & 0,16 \\
\hline No & 152582 & 1,00 & & & 0,07 & 0,05 & 0,08 \\
\hline \multicolumn{8}{|l|}{ Covariables $^{1}$} \\
\hline Periodo de secado previo (días) & & 0,99 & 0,98 & 1,00 & & & \\
\hline Duración de lactancia previa (días) & & 1,04 & 1,03 & 1,05 & & & \\
\hline Producción en lactancia previa (kg) & & 1,17 & 1,15 & 1,19 & & & \\
\hline
\end{tabular}

1 Los OR reportados están calculados para incrementos de 30 d sobre el promedio de periodo de secado (x=83 d) y duración de lactancia ( $\mathrm{x}=311 \mathrm{~d})$; y $1000 \mathrm{~kg}$ en producción de leche $(\mathrm{x}=5750 \mathrm{~kg})$. 
significativa para el modelo alternativo, sí lo fue dentro del modelo base, y las tendencias en los OR y PM mostraron patrones concordantes en ambos modelos, con una marcada mayor propensión para la zona bs_t y menor para bh_t, y las demás zonas no difieren significativamente entre sí. Cabe señalar que para la zona bs_t se contó con 931 lactancias provenientes de solo 4 hatos, uno de los cuales es de Doble Propósito. Esto se refleja en la amplitud del intervalo de confianza $(2,3-55,8)$ por lo que el OR tiene un alto margen de error. La mayor propensión a mastitis en esta zona podría estar ligada a las altas temperaturas características de esta zona, que podrían facilitar la mayor proliferación de bacterias causantes de esta enfermedad.

Con respecto al número de parto (Cuadro 2) se observa que las vacas más propensas a mastitis fueron aquellas con 4 partos, mientras que las menos propensas fueron las de 6 o más partos. No obstante se debe tener en cuenta que en el modelo alternativo no se incluyen las vacas de primer parto, que es precisamente el grupo menos propenso de acuerdo con los resultados del modelo base. Las probabilidades marginales de mastitis oscilaron desde 0,03 (primer parto, del modelo base) hasta 0,08 (partos 3,4 y 5; modelo alternativo). En ambos modelos, la probabilidad de contraer mastitis aumenta de manera consistente hasta los partos 4 y 5 , después de lo cual tiende a estabilizarse. Steeneveld et ál. (2008) también reportaron una menor incidencia de mastitis en novillas de primer parto en comparación con vacas multíparas. Novoa et ál. (2005) reportaron mayor incidencia de mastitis en vacas de mayor edad y mayor número de partos.

En cuanto a los meses de parto (Cuadro 3), se observó que las vacas que paren en marzo, abril y mayo son las de mayor propensión a presentar mastitis, mientras que las que paren en Octubre y Noviembre son menos propensas. Las probabilidades marginales de mastitis oscilaron entre 0,06 (Octubre y Noviembre) y 0,08 (Febrero hasta Mayo). Estos 3 meses donde hubo mayor prevalencia de mastitis son los más calientes del año y corresponden a la época seca. Al existir altas temperaturas, puede haber mayor proliferación de bacterias causantes de esta enfermedad. Los efectos estacionales han sido significativos en algunos estudios, pero dependen de la latitud y condiciones de alojamiento. Elbably et ál. (2013) reportaron una mayor prevalencia de mastitis durante el verano, mientras que Novoa et ál. (2005) reportaron mayores prevalencias en el periodo lluvioso. Moosavi et ál. (2014) reportaron mayor incidencia de mastitis en etapas tempranas de lactancia durante invierno y primavera, y mayor incidencia de mastitis en etapas tardías de la lactancia durante el verano. Las influencias estacionales son más evidentes en sistemas de producción bajo pastoreo en comparación con sistemas estabulados, si bien las tasas de infección tienden a ser más bajas en pastoreo por el menor contacto entre animales y por ser un ambiente relativamente más limpio (Berry and Meany 2005).

En términos de periodo de la lactancia la propensión a primeros eventos de mastitis fue acentuadamente mayor en el primer mes posparto. La reducción en la propensión es consistente conforme aumentan los días posparto, mientras que tiende a estabilizarse después del día 300. Las probabilidades marginales de mastitis oscilaron entre 0,02 (a partir del día 270 posparto) y 0,63 (primer mes posparto). Otros estudios también reportan un mayor riesgo de contraer mastitis durante los primeros días de lactancia (Suriyasathaporn et ál. 2000, Berry y Meany 2005, Biffa et ál. 2005, Steeneveld et ál. 2008, Elbably et ál. 2013). La primera fase de la lactancia es, sin duda alguna, el momento de mayor estrés para la vaca lechera ya que se produce un incremento marcado en producción de leche en un tiempo muy reducido, lo que sin duda contribuye a una mayor susceptibilidad a las infecciones mamarias.

Entre los factores relacionados con la lactancia previa (Cuadro 3) se observó que las vacas que fueron positivas para mastitis en la lactancia anterior fueron 2,1 veces más propensas de contraer mastitis en la presente lactancia. Estos resultados son similares a los obtenidos por Berry and Meany (2005), Biffa et ál. (2005), Pinedo et ál. (2012) y Pantoja et ál. (2009), quienes observaron 
que los animales con casos de mastitis en la lactancia previa fueron 2,$02 ; 4,2 ; 5$ y 4,1 veces, respectivamente, más susceptibles a presentar la enfermedad en la siguiente lactancia. Algunos tipos de mastitis, particularmente las de origen infeccioso, pueden prevalecer a lo largo de una o varias lactancias.

Con respecto a las covariables (Cuadro 3) se observó que un incremento de 30 días sobre el promedio de duración de la lactancia previa se asoció con una mayor propensión a mastitis en la lactancia presente (OR 1,04). Lactancias extensas pueden estar ligadas a deficiencias en el secado, con la consecuente mala preparación de la ubre para la lactancia siguiente, lo que podría promover la aparición de mastitis. De modo similar se observó que un incremento en producción de $1000 \mathrm{~kg}$ por lactancia sobre el promedio también se asoció con un incremento marcado en la propensión a contraer mastitis (Cuadro 3, OR 1,17). Lo anterior concuerda con los resultados encontrados por Elbably et ál. (2013), quienes concluyeron que existe una alta relación entre la producción de leche y la ocurrencia de mastitis. Se han encontrado además correlaciones genéticas desfavorables entre producción de leche y mastitis (Oltenacu y Broom 2010).

Los resultados de este estudio demuestran la importancia de factores ligados a la vaca y su entorno en la frecuencia de eventos de mastitis dentro de los hatos lecheros. La incidencia observada de mastitis clínica, aunque obtenida a partir de registros de hato, se encuentra dentro de los rangos reportados por otros estudios. El tipo racial, la zona ecológica de vida, el mes y año de parto, el número de partos, el periodo de la lactancia, la presencia de casos previos y la producción de leche fueron confirmados como factores de riesgo para el desarrollo de la mastitis clínica en los hatos lecheros de Costa Rica. Estos hallazgos pueden ser útiles para el desarrollo de protocolos preventivos enfocados a reducir la incidencia de mastitis en los grupos de mayor riesgo.

\section{LITERATURA CITADA}

ALMAW G., MOLLA W., MELAKU A. 2012. Incidence rate of clinical bovine mastitis in selected smallholder dairy farms in Gondar town, Ethiopia. Ethiopian Veterinary Journal 16(1):93-99.

ANDRESEN H. 2001. Mastitis, prevención y control. Revista de Investigaciones Veterinarias del Perú 12(2):55-64.

BERRY D.P., MEANEY W.J. 2005. Cow factors affecting the risk of clinical mastitis. Irish Journal of Agricultural and Food Research 44(1):147-156.

BIFFA D., DEBELA E., BEYENE F. 2005. Prevalence and Risk Factors of Mastitis in Lactating Dairy Cows in Southern Ethiopia. International Journal of Applied Research in Veterinary Medicine 3:189-198.

BLOWEY R.W., EDMONDSON P. 2010. Mastitis Control in Dairy Herds. 2 ed. Oxfordshire, UK. CAB International. $266 \mathrm{p}$.

COOPERATIVA DE PRODUCTORES DE LECHE DOS PINOS R.L. 2010. Reglamento de Recibo de Leche. $26 \mathrm{p}$.

ELBABLY M.A., EMEASH H.H., ASMAA N.M. 2013. Risk Factors Associated with Mastitis Occurrence in Dairy Herds in Benisuef, Egypt. World's Veterinary Journal 3(1):5-10.

GIANNEECHINI R., CONCHA C., RIVERO R., DELUCCI I., MORENO J. 2002. Occurrence of clinical and sub-clinical mastitis in dairy herds in the west littoral region in Uruguay. Acta Veterinaria Scandinavica 43(1):221-230.

HOLDRIDGE L. 1987. Ecología basada en zonas de vida. Traducido por Humberto Jiménez Saa. San José, Costa Rica. IICA. 216 p

KLEINBAUM D.G., KLEIN M. 2010. Logistic regression a self-learning text. Statistics for biology and health. 3rd edition. Springer editorial. New York, USA. $709 \mathrm{p}$.

MEKONNEN G., BERIHUN A., HABTAMU T. 2012. Prevalencia de mastitis y su relación con factores de riesgo en granjas lecheras de pequeños propietarios en Mekelle y sus alrededores. Revista Electrónica de Veterinaria 13(9). Disponible en http://www. veterinaria.org/revistas/redvet/n090912.html

MOOSAVI M., MIRZAEI A., GHAVAMI M., TAMADON A. 2014. Relationship between season, lactation number and incidence of clinical mastitis in different stages of lactation in a Holstein dairy farm. Veterinary Research Forum 5(1):13-19.

NIELSEN C. 2009. Economic Impact of Mastitis in Dairy Cows. Tesis Doctoral. Uppsala, Suecia, Swedish University of Agricultural Sciences. $81 \mathrm{p}$. 
NOORDHUIZEN J.P.T.M., BUURMAN J. 1984. VAMPP: A Veterinary automated management and production control program for dairy farms. The application of MUMPS for data processing. Veterinary Quarterly 6(2):66-72.

NOVOA R., ARMENTEROS M., ABELEDO M.A., CASANOVAS E., VALERA R., CABALLERO C., PULIDO J. 2005. Factores de riesgo asociados a la prevalencia de mastitis clínica y subclínica. Revista Salud Animal 27(2):84-88.

OLDE RIEKERINK R.M., BARKEMA H., STRYHN H. 2007. The Effect of Season on Somatic Cell Count and the Incidence of Clinical Mastitis. Journal of Dairy Science 90(4):1704-1715.

OLTENACU P.A., BROOM D.M. 2010. The impact of genetic selection for increased milk yield on the welfare of dairy cows. Animal Welfare 19(S):39-49.

PANTOJA J.C.F., HULLAND C., RUEGG P.L. 2009. Somatic cell count status across the dry period as a risk factor for the development of clinical mastitis in the subsequent lactation Journal of Dairy Science 92(1):139-148.

PÉREZ E., BAAYEN M.T., CAPELLA E., BARKEMA H. 1989. Development of a livestock information system for Costa Rica, pp. 221-224. In: H. Kuil, R.W. Palin and J.E. Huhn (eds.). Livestock Production and Diseases in the Tropics. Proceedings 4th International Conference Inst. Trop. Vet. Med. Utrecht; The Netherlands.

PINEDO P.J., FLEMING C., RISCO C.A. 2012. Events occurring during the previous lactation, the dry period, and peripartum as risk factors for early lactation mastitis in cows receiving 2 different intramammary dry cow therapies. Journal of Dairy Science 95(12):7015-7026.

PRENDIVILLE R., PIERCE K.M., BUCKLEY F. 2010. A comparison between Holstein-Friesian and Jersey dairy cows and their F1 cross with regard to milk yield, somatic cell score, mastitis, and milking characteristics under grazing conditions. Journal of Dairy Science 93(6):2741-2750.

QUESADA C. 1994. Incidencia de mastitis subclínica en seis fincas de la zona de San Carlos. Práctica de Especialidad presentada para obtener el grado de Bachiller en Ingeniería Agronómica, Instituto Tecnológico de Costa Rica, San Carlos, Costa Rica. $76 \mathrm{p}$.
RAHMAN M.A., BHUIYAN M.M.U., KAMAL M.M., SHAMSUDDIN M. 2009. Prevalence and risk factors of mastitis in dairy cows. The Bangladesh Veterinarian 26(2):54-60.

RAMÍREZ N., ARROYAVE O., CERÓN M., JARAMILLO M., CERÓN J., PALACIO G. 2011. Factores asociados a mastitis en vacas de la microcuenca lechera del altiplano norte de Antioquia, Colombia. Revista Medicina Veterinaria 22:31-42.

RICHERT R.M., CICCONI K.M., GAMROTH M.J., SCHUKKEN Y.H., STIGLBAUER K.E., RUEGG P.L. 2013. Risk factors for clinical mastitis, ketosis, and pneumonia in dairy cattle on organic and small conventional farms in the United States. Journal of Dairy Science 96(7):4269-4285.

ROTHMAN K.J., GREENLAND S., LASH T.L. 2008. Modern Epidemiology, 3rd Edition. Philadelphia, PA: Lippincott, Williams \& Wilkins. 758 p.

RUPP R., BOCCHARD D. 2004. Genetics of resistance to mastitis in dairy cattle. Veterinary Research 34(5):671-688.

SANTIVAÑEZ C.S., GÓMEZ O.E., CÁRDENAS L.A., ESCOBEDO M.H., BUSTINZA R.H., PEÑA J. 2013. Prevalencia y factores asociados a la mastitis subclínica bovina en los Andes peruanos. Veterinaria y Zootecnia 7(2):92-104.

SAS INST. INC. 2009. SAS/STAT® User's guide: Statistics; Version 9.3. Cary; NC, USA. 8640 p.

SCHUKKEN Y.H., BARB D., HERTL J., GRÖHN Y.T 2010. Correlated time to event data: Modeling repeated clinical mastitis data from dairy cattle in New York State. Preventive Veterinary Medicine 97(3):150-156.

STEENEVELD W., HOGEVEEN H., BARKEMA H.W., VAN DEN BROEK J., HUIRNE R.B.M. 2008. The Influence of Cow Factors on the Incidence of Clinical Mastitis in Dairy Cows. Journal of Dairy Science 91(4):1391-1402.

SURIYASATHAPORN W., SCHUKEN Y.H., NIELEN M., BRAND A. 2000. Low Somatic Cell Count: a Risk Factor for Subsequent Clinical Mastitis in a Dairy Herd. Journal of Dairy Science 83(6):1248-1255.

VARGAS B., SOLÍS O., SÁENZ F., LEÓN H. 2013. Análisis multivariado para caracterización y clasificación de hatos lecheros en Costa Rica. Agronomía Mesoamericana 24(2):257-275.

Todos los derechos reservados. Universidad de Costa Rica. Este artículo se encuentra licenciado con Creative Commons Reconocimiento-NoComercial-SinObraDerivada 3.0 Costa Rica. Para mayor información escribir a rac.cia@ucr.ac.cr 
